\title{
Coronary Spasm due to Type A Aortic Dissection Complicated by Hemopericardium: A Case Report of Another Possible Cause of Coronary Malperfusion
}

\author{
Noppadol Chamnarnphol, Sirichai Cheewatanakornkul and Treechada Wisaratapong
}

\begin{abstract}
Coronary malperfusion complicating Type A aortic dissection is relatively rare. The diagnosis of Type A aortic dissection as the cause of coronary ischemia is a challenge. The mechanism of coronary malperfusion has been proposed. We report a 45-year-old man presenting with acute inferior wall ST segment elevation myocardial infarction who was finally diagnosed to have Type A aortic dissection complicated by hemopericardium and cardiac tamponade. Coronary spasm is the most likely cause of transient myocardial ischemia in this patient and should be considered as another possible cause of coronary malperfusion in patients with Type A aortic dissection.
\end{abstract}

Key words: aortic dissection, hemopericardium, acute myocardial infarction, coronary spasm

(Inter Med 49: 829-831, 2010)

(DOI: 10.2169/internalmedicine.49.3192)

\section{Introduction}

Type A aortic dissection is one of the most catastrophic illnesses. Major complications include aortic rupture with or without cardiac tamponade, acute aortic regurgitation and compromised blood flow to aortic branches (1). Coronary ischemia related to aortic dissection is relatively rare, but once it occurs, it is fatal $(2,3)$. Understanding the mechanism of coronary malperfusion can facilitate the most appropriate treatment. The purpose of this report is to describe another possible mechanism of coronary malperfusion in a patient with type A aortic dissection with hemopericardium.

\section{Case Report}

A 45-year-old man with untreated hypertension and a history of heavy smoking was referred to our hospital because of the acute onset of severe chest pain and shock. His blood pressure at the referral hospital was $90 / 60 \mathrm{mmHg}$ and pulse rate was $110 / \mathrm{min}$. Physical examination revealed good consciousness, tachypnea and dyspnea with sweating, no mur- mur, clear lung field and equal peripheral pulses.

An electrocardiogram showed sinus tachycardia with ST segment elevation in leads II, III, and ${ }_{a} \mathrm{~V}_{\mathrm{F}}$ and ST depression in leads $\mathrm{I},{ }_{\mathrm{a}} \mathrm{V}_{\mathrm{L}}$ and $\mathrm{V}_{4}-\mathrm{V}_{5}$. The right precordial ECG was not done. He was diagnosed as acute inferior ST-segment elevation myocardial infarction with suspected right ventricular infarction and cardiogenic shock. Aspirin (300 mg), 6,000 units of unfractionated heparin and $500 \mathrm{~mL}$ of normal saline were promptly given.

At our hospital, his blood pressure was $120 / 80 \mathrm{mmHg}$, pulse rate was $120 / \mathrm{min}$ and respiratory rate was $28 / \mathrm{min}$. His chest pain partially subsided and the repeat ECG (approximately 90 minutes after the first ECG) revealed sinus tachycardia with no evidence of ST segment elevation or depression. However, the patient was taken to the cardiac catheterization laboratory as it is the usual procedure for emergency coronary angiography (CAG) and primary percutaneous coronary intervention (PPCI). Transthoracic echocardiography (TTE) is definitely useful to detect complication of AMI and also other causes of chest pain. However, we do not routinely perform TTE before PPCI so as to keep the door-to-balloon time as short as possible.

Division of Cardiovascular Medicine, Department of Internal Medicine, Faculty of Medicine, Prince of Songkla University, Hadyai, Songkhla, Thailand

Received for publication November 26, 2009; Accepted for publication December 21, 2009

Correspondence to Dr. Noppadol Chamnarnphol, cnoppado@hotmail.com 

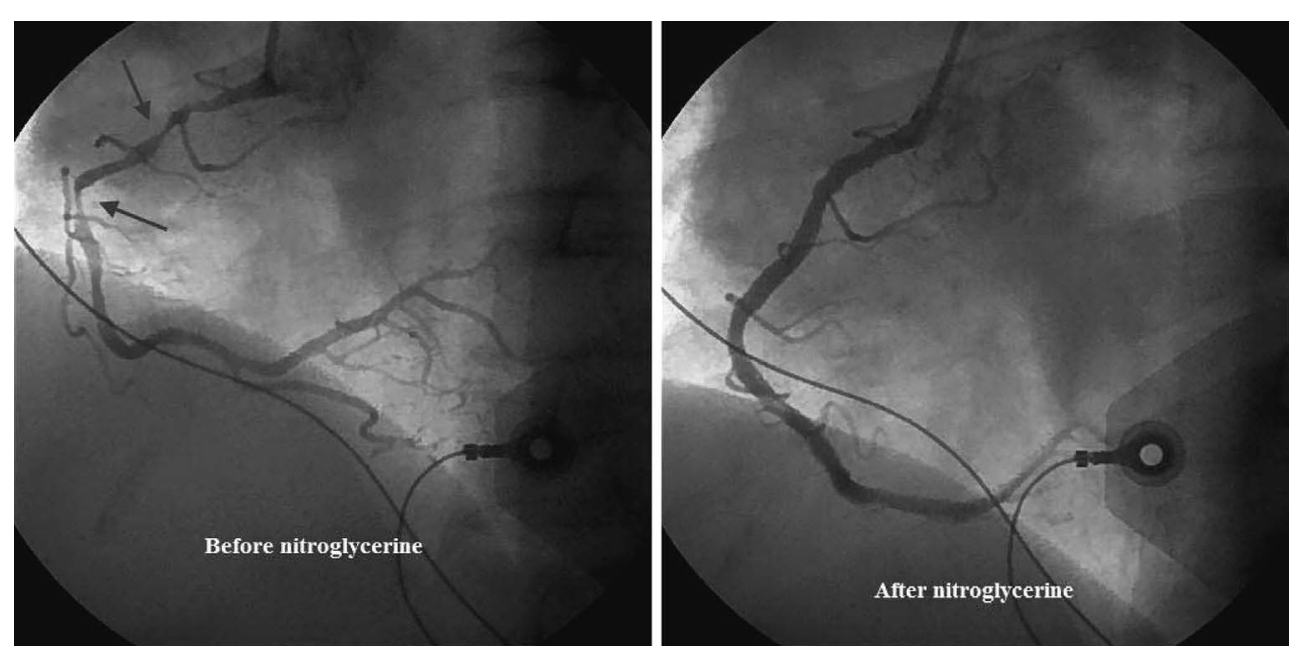

Figure 1. (A) Severe stenosis in the proximal and mid RCA before IC nitroglycerin and (B) after IC nitroglycerin.

Emergency CAG was done as the usual technique. Engaging the ostial right coronary artery (RCA) was performed smoothly. There was some difficulty to engage the left coronary artery (LCA) as the Judkins left diagnostic catheter could not move freely in the ascending aorta. However, it was not remarkable to the operator at that time. CAG revealed $90 \%$ stenosis without intraluminal thrombus in the proximal and mid RCA followed by diffusely small diameter of the distal RCA. The ostial RCA was normal. The LCA had no significant flow-limiting disease. After a 100 microgram of intracoronary (IC) nitroglycerin injection, the severe stenosis in the proximal RCA disappeared and the mid- to distal parts markedly dilated (Fig. 1). Left ventriculography using $10 \mathrm{~mL}$ of diluted contrast media via hand injection showed hyperkinetic left ventricular contraction without evidence of wall motion abnormality. Due to the small volume of contrast media, the ascending aorta was not well opacified. There was no stagnation of contrast media in the aorta after the procedure. The left ventricular end diastolic pressure (LVEDP) was $17 \mathrm{mmHg}$.

After CAG, despite the lack of evidence of residual ST segment elevation on the subsequent 12-lead ECG, ongoing chest discomfort, tachypnea and tachycardia remained. The patient's systolic blood pressures ranged from 100-120 $\mathrm{mmHg}$, which was much lower than his usual blood pressure. A TTE (Fig. 2) was then performed which demonstrated an intimal flap in the ascending aorta just above the ostial RCA in diastolic phase. There was a moderate amount of pericardial effusion with right ventricular (RV) diastolic collapse, albeit preserved RV contractility, compatible with type A aortic dissection with hemopericardium and cardiac tamponade. To confirm the diagnosis as well as to identify the disease extension, the patient underwent emergency computed tomography (CT) scan of the chest which confirmed type A aortic dissection with hemopericardium and the intimal flap extended to the aortic arch. There was also evidence of active extravasation of contrast media from the ascending aorta into pericardial space, a moderate amount of

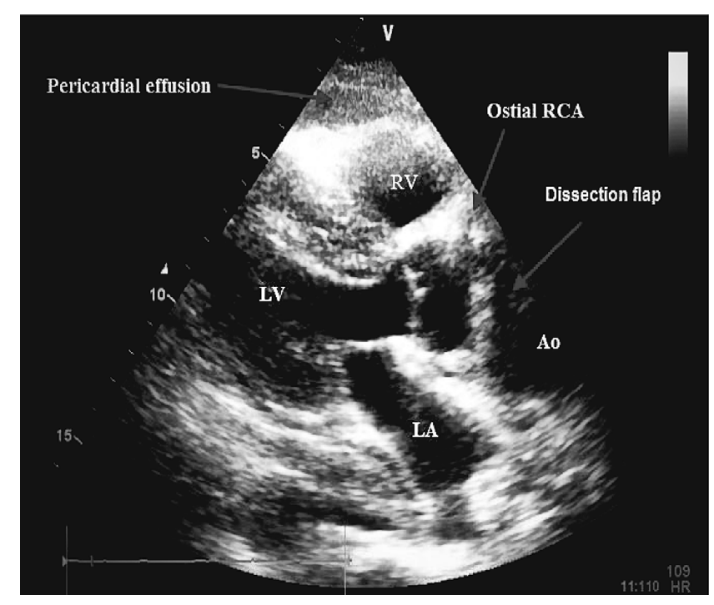

Figure 2. Transthorasic echocardiography (TTE) demonstrated an aortic dissection flap just above the patent ostial RCA with pericardial effusion anterior to the right ventricle (RV).

pericardial effusion and right hemothorax.

Emergency surgery was successfully performed. The operative findings were type A aortic dissection with hemopericardium and the intimal flap extended to the aortic arch. The ostial RCA and aortic valve were not involved by the dissection. The postoperative period was uneventful and the patient was discharged on the 10th postoperative day. At the 12-month follow-up, he remained free of symptoms with a normal ECG.

\section{Discussion}

End-organ ischemia is one of the major complications of aortic dissection as the aorta gives rise to many important branches including the coronary artery (1). Most type A dissections arise above the right sinus of Valsalva and retrograde extension into the aortic root more often affects the right coronary artery (4). Unfortunately, diagnosis of type A aortic dissection as the cause of coronary malperfusion is difficult and may be unrecognized. The consequences of 
such a misdiagnosis in the era of thrombolysis can be catastrophic (4).

The branch arteries may become poorly perfused as a result of fixed obstruction to their origin or dynamic obstruction from dynamic changes in the true or false lumen (2). According to the Neri's definition of coronary malperfusion in acute aortic dissection: Type $\mathrm{A}$ is a coronary occlusion resulting from compression by the bulging dissected false lumen or by secondary extravasation of blood into the pericardial or perivascular tissues (5). Type B is a retrograde extension of the dissection into the coronary arterial wall. Type $\mathrm{C}$ is the coronary artery detachment from the aortic root.

In the present case, the initial ECG demonstrated STE in inferior leads followed by spontaneous resolution in the second ECG. The mechanisms that may explain spontaneous STE resolution included dynamic obstruction by the bulging of false lumen or prolapse of unsupported intimal flap which may transiently obstructed the ostial RCA. This mechanism was type A Neri's classification which must occur transiently to account for STE reversibility. The Neri classification type B and C cannot explain spontaneous STsegment resolution.

The more likely cause of transient ST-segment elevation in this case was coronary spasm which was demonstrated by coronary angiography. The catheter-induced coronary spasm was unlikely because the patency of ostial RCA was spared and the spasm was located quite far away from the catheter tip. Coronary spasm may be related to hemopericardium.
Blood-bathed coronary artery could develop spasm as hemoglobin may interfere with nitric oxide action and inhibit endothelium-dependent relaxation (6). The vasospasm phenomenon occurs rarely only in special circumstances, as in this case, but it is very common in the clinical context of cerebral vasospasm due to subarachnoid hemorrhage.

Missed diagnosis of aortic dissection may result in emergency PCI and insertion of intra-aortic balloon pump (IABP). Both procedures can harm the patient as post PCI patient requires dual antiplatelets to prevent stent thrombosis. Discontinuing dual antiplatelets after surgery for aortic dissection may result in stent thrombosis. In contrast, continuing dual antiplatelets may cause peri-operative bleeding. Moreover, IABP may worsen the aortic dissection. Spontaneous resolution of ST segment is an important clue of coronary spasm and contemplation of injection of intracoronary nitroglycerin to distinguish coronary spasm and fixed stenosis is crucial.

\section{Conclusion}

The authors reported a patient with type A aortic dissection complicated by hemopericardium, cardiac tamponade and coronary spasm. The patient presented with acute inferior STE myocardial infarction and shock. Coronary spasm is another mechanism of coronary malperfusion in a patient with type A aortic dissection complicated by hemopericardium.

\section{References}

1. Golledge J, Eagle KA. Aortic dissection, Seminar. Lancet 372: 5566, 2008.

2. Kawahito K, Adachi H, Murata S, Yamaguchi A, Ino T. Coronary malperfusion due to type A aortic dissection: mechanism and surgical management. Ann Thorac Surg 76: 1471-1476, 2003.

3. Hirst AE, Johns VJ, Kime SW. Dissecting aneurysms of the aorta: a review of 505 cases. Medicine 37: 217-271, 1958.

4. Isselbacher EM. Disease of the aorta. In: Braunwald's Heart Disease, A Textbook of Cardiovascular Medicine. 7th ed. Zipes DP,
Libby P, Bonow RO, Braunwald E, Eds. Saunders, Philadelphia, 2005: 1403-1435.

5. Neri E, Toscano T, Papalia U, et al. Proximal aortic dissection with coronary malperfusion: presentation, management, and outcome. J Thorac Cardiovasc Surg 121: 552-560, 2001.

6. Collins P, Burman J, Chung HI, Fox K. Hemoglobin inhibits endothelium dependent relaxation to acetylcholine in human coronary arteries in vivo. Circulation 87: 80-85, 1993.

(C) 2010 The Japanese Society of Internal Medicine http://www.naika.or.jp/imindex.html 